Supporting Information

\title{
Fucoidan hydrogels photocrosslinked with visible radiation as matrices for cell culture
}

Lara L. Reys $*^{\dagger,}$, Simone S. Silva ${ }^{\dagger}+$, Diana Soares da Costa ${ }^{\dagger,}$, Nuno M. Oliveira ${ }^{\dagger}$, João F. Mano $^{\dagger, \ddagger}$, Rui L. Reis ${ }^{\dagger, *}$ and Tiago H. Silva ${ }^{* \dagger, *}$

†3B’s Research Group - Biomaterials, Biodegradables and Biomimetics, University of Minho, Headquarters of the European Institute of Excellence on Tissue Engineering and Regenerative Medicine, AvePark- Parque de Ciência e Tecnologia, 4805-017 Barco, Guimarães, Portugal. ${ }^{\ddagger} \mathrm{ICVS} / 3 \mathrm{~B}$ 's - PT Government Associated Laboratory, Braga/Guimarães, Portugal.

\section{1 - Classical strategies for chemical crosslinking of fucoidan}

The high solubility of fucoidan in aqueous solution hinders the formation of polymeric matrices structurally stable in such media, from which crosslinking strategies are deemed as necessary. Reaction of fucoidan with different crosslinking agents, namely epichlorohydrin or 1,4 Butanediol diglycidyl ether (BDDE) to address polymer crosslinking, was assessed, similar to what have been successfully accomplished with other sulfated polysaccharides, namely carrageenan and ulvan, respectively. Fucoidan was combined with epichlorohydrin at $4.63 \mathrm{mM}$ in water solution of $50 \%(\mathrm{v} / \mathrm{v})$ 2-propanol, $0.32 \mathrm{M} \mathrm{KOH}$ at $37^{\circ} \mathrm{C}, 100 \mathrm{rpm}$ for $24 \mathrm{~h}$ and the crosslinking reaction resulted in the formation of flake-like agglomerates. Such precipitate could not be dissolved in water and other solvents, namely DMSO $(1 \% \mathrm{v} / \mathrm{v}), \mathrm{NaOH} 1 \mathrm{M}$ and acetic acid $2 \%(\mathrm{v} / \mathrm{v})$ (Figure $\mathrm{S} 1$ ), neither be processed into polymeric structures with defined morphology. Fucoidan $(4$ and $8 \% \mathrm{w} / \mathrm{v})$ was also combined with BDDE in different concentrations from 0.20 to $2.00(\mathrm{w} / \mathrm{w})$ in proportion of crosslinker relatively to fucoidan at room temperature at $100 \mathrm{rpm}$ 
in period of time between 15 minutes to 24 hours, but the crosslinking reaction was not effective, with the resulting precipitate being still highly soluble in water.

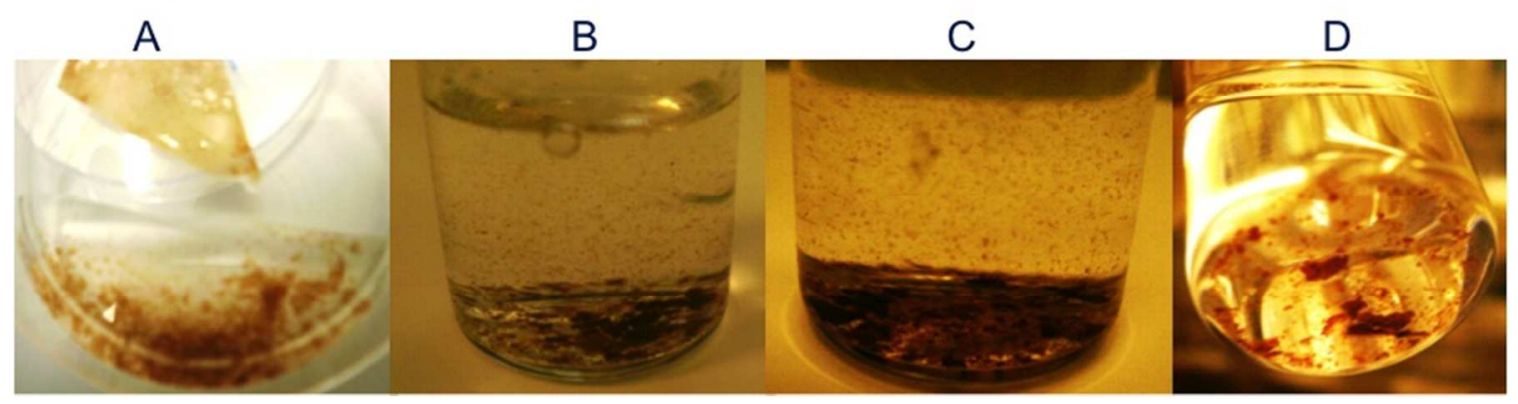

Figure S1: Immersion of fucoidan cross-linked with epichlorohydrin in water (A) and different solvents (B- in $\mathrm{NaOH} 2 \mathrm{M}, \mathrm{C}$ - in acetic acid and D- in DMSO).

\section{2 - Evaluation of the structural stability of fucoidan-based particles in aqueous solution}

Modified fucoidan-based particles were successfully formed (Figure S2 A) onto superhydrophobic surfaces and photocrosslinked using eosin-y and triethanolamine (TEOA) as photoiniciators upon the irradiation with visible light, following the procedure described in the experimental section of the manuscript. The structural stability of the produced fucoidan-based particles in aqueous solution was assessed by incubation in Dulbecco's phosphate buffered saline (DPBS), at $37^{\circ} \mathrm{C}$ and $60 \mathrm{rpm}$, during fifteen days (Figure S2 B). The produced particles revealed to be stable, with their structure remaining constant all long the period of study. 


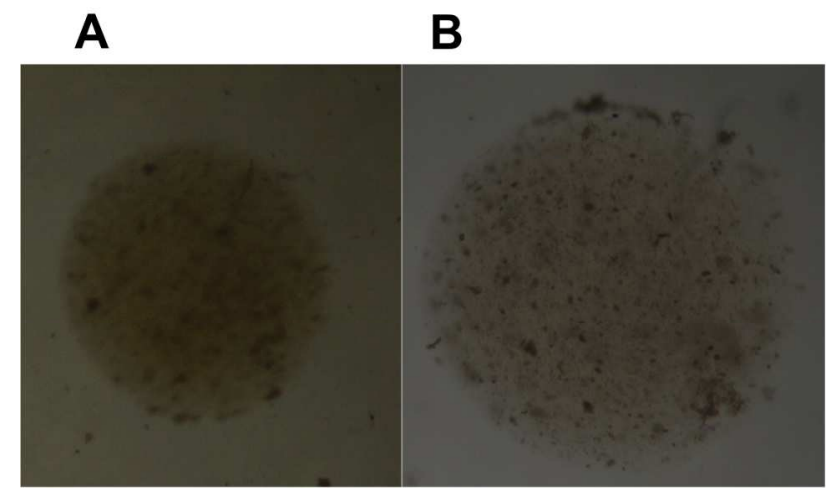

Figure S2: Immersion of fucoidan based particles in DPBS in different time point's A) After immersion (day 0) and B) after 15 days of immersion. 